\title{
Quantized Transport in Two-Dimensional Spin-Ordered Structures
}

\author{
Ilaria Campana ${ }^{1}$, Giancarlo $\mathrm{Jug}^{2}$ and Klaus Ziegler ${ }^{1}$ \\ ${ }^{1}$ Institut für Physik, Universität Augsburg, D-86135 Augsburg (Germany) \\ ${ }^{2}$ Dipartimento di Fisica e Matematica, Universitá dell’Insubria, 22100 Como (Italy)
}

16th November 2018

\begin{abstract}
We study in detail the transport properties of a model of conducting electrons in the presence of double-exchange between localized spins arranged on a 2D Kagome lattice, as introduced by Ohgushi, Murakami and Nagaosa (2000). The relationship between the canting angle of the spin texture $\theta$ and the Berry phase field flux per triangular plaquette $\phi$ is derived explicitly and we emphasize the similarities between this model and Haldane's honeycomb lattice version of the quantum Hall effect (Haldane, 1988). The quantization of the transverse (Hall) conductivity $\sigma_{x y}$ is derived explicitly from the Kubo formula and a direct calculation of the longitudinal conductivity $\sigma_{x x}$ shows the existence of a metal-insulator transition as a function of the canting angle $\theta$ (or flux density $\phi$ ). This transition might be linked to that observable in the manganite compounds or in the pyrochlore ones, as the spin ordering changes from ferromagnetic to canted.
\end{abstract}

\section{Introduction}

A fascinating and relatively common problem in solid-state physics is the motion of electrons in a lattice structure where localized spins are present, possibly in an organised form. The scattering of the electrons by the spins represents a complex physics problem for the theoretical investigator, especially when (like it seems to happen for the high- $T_{c}$ cuprate superconductors) the spin and charge degrees of freedom are attached to the same particles - a situation which we do not consider. In a simplified picture we assume first of all that the spins are localised and interacting, but that the mobile electrons do not interact with each other (or interact weakly and give rise to independent quasiparticles) and are separate entities from the electrons that produce the magnetic ions. In this case the individual electrons (or quasiparticles) experience the localized spins as an effective local magnetic flux (Müller-Hartmann and Dagotto, 1996). This flux produces a Berry (or Peierls) phase in the hopping terms of the electrons' Hamiltonian. In this paper we shall study a situation with a locally staggered flux, created by plaquettes of ordered spins, where the global flux is zero. This case is realized, for instance, in a Kagome lattice (Ohgushi, Murakami and Nagaosa, 2000), a two dimensional (2D) lattice consisting of triangles and hexagons of the same interatomic distance and that can be viewed as a triangular Bravais lattice with a three-point atomic basis forming an equilateral triangle of size half that of the triangular lattice parameter's. In Fig.1 we remind the reader of the Kagome lattice structure.

This type of lattice may have experimental relevance in the planes of pyrochlore compounds (Ohgushi, Murakami and Nagaosa, 2000, Ramirez, 1994, Harris and Zinkin, 1996) and the transport properties we describe may be appropriate for such materials. Ferromagnetic pyrochlore crystals of the type $\mathrm{R}_{2} \mathrm{Mo}_{2} \mathrm{O}_{7}$ $(\mathrm{R}=\mathrm{Nd}, \mathrm{Sm}$ or $\mathrm{Gd})$ have revealed interesting transport properties like an anomalous Hall effect increasing as the temperature $T$ is lowered, (Taguchi and Tokura, 1999) a feature that seems to be connected with the geometrical frustration of pyrochlore lattices that is partly embodied by the Kagome lattice itself, viewed now as the $(1,1,1)$ cross-section of the 3D pyrochlore's lattice. Motivated by the transport properties of pyrochlore compounds, as well as by those of the manganite ones, we study in this paper some quantum transport properties of the Kagome lattice with a canted localised spin texture in which independent electrons can move. 


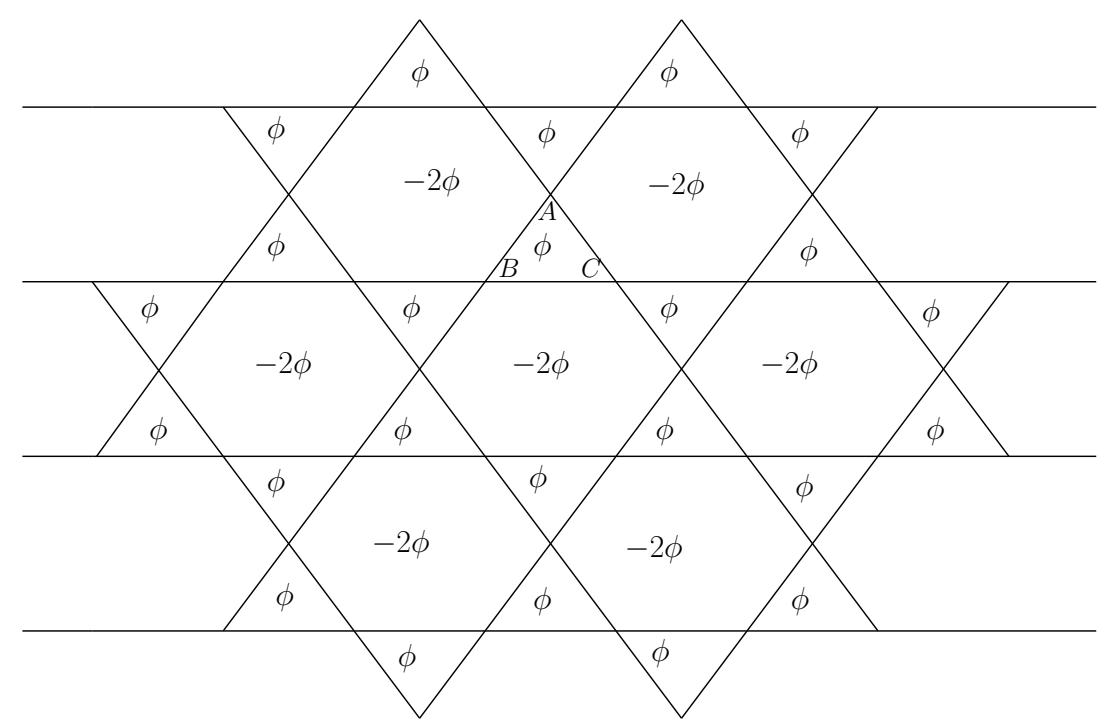

Figure 1: Kagome lattice

The behavior of the Berry phase as a function of the spin canting for the model at hand, and its consequences in terms of the macroscopic transport properties of the model are studied in this paper. We discuss in detail the energy spectrum as it depends strongly on the canting of the localized spins. In particular, the nodes in the spectrum and the opening of energy gaps are investigated, including their consequences for the transport properties. Moreover, we evaluate explicitely the longitudinal conductivity $\sigma_{x x}$ and the Hall conductivity $\sigma_{x y}$ as a function of the canting angle $\theta$ or flux $\phi$. Our results for the transport properties are then compared with those of another famous model of this class, where a staggered magnetic field is applied to electrons within a honeycomb lattice (Haldane, 1988). The latter has very similar spectral properties as the model, first proposed by Ohgushi, Murakami and Nagaosa, (2000) defined on the Kagome lattice and studied here in greater detail. The model on the honeycomb lattice was proposed by Haldane as the condensed-matter (solid-state) equivalent of the quantum Hall effect, in that a quantization of the Hall $\sigma_{x y}$ conductivity can be achieved by varying the local flux per plaquette $\phi$, but without the need to introduce an external, homogeneous magnetic field. In the case of the Kagome lattice model at hand, the same result will be shown to be attained through the introduction of a localised spin texture. Our results confirm and complete the work by Ohgushi, Murakami and Nagaosa for the Hall $\sigma_{x y}$ conductivity, with explicit calculations in terms of expansions around the gap's nodes shown in detail, and moreover the quantized values of the longitudinal $\sigma_{x x}$ conductivity are obtained indicating the existence of some sort of metal-insulator transition as the canting angle moves away from some special values.

The paper is organized as follows: In Section 2 the tight-binding model of Ohgushi, Murakami and Nagaosa for localized spins is described. The relationship between the localized spin's wavefunction and the Berry phase of the hopping elctron is discussed in Section 2.1, and the application to the Kagome lattice (Section 2.2) is presented. In Section 2.3 the tigh-binding model for the honeycomb lattice with a staggered magnetic field is also presented and compared to the model on the Kagome lattice. Transport properties are then studied in Section 3 by making explicit use of Kubo's formula and an expansion of the energy spectrum near the nodes next to the Fermi energy, and the results obtained are discussed in Section 4 . 


\section{A Model of Hopping Electrons in a Spin Texture}

\subsection{The Model}

We consider the electronic hopping between nearest neighbours on a Kagome lattice as described by the tight-binding Hamiltonian. The electronic degrees of freedom are coupled to a set of localized spin- $S$ degrees of freedom on the same lattice via a local Hund coupling $J_{H}$. In this work $S=\frac{1}{2}$, but generalization to the physically and theoretically interesting case of larger $S$ is possible. When $J_{H}$ is strong enough the spin of the hopping electron is forced to allign parallel to the localised spin $\mathbf{S}_{i}$ at each site and through double-exchange mechanism (Zener, 1951, Anderson and Hasegawa, 1955, de Gennes, 1960 ) the tight-binding hopping parameter $t_{i j}$ between two neighbouring sites $\langle i, j\rangle$ becomes proportional to the projection of the localised-spin wave function at site $j$ onto that at site $i$. The effective Hamiltonian representing the hopping is then

$$
H=\sum_{\langle i, j\rangle} t_{i j}^{e f f} c_{i}^{\dagger} c_{j}+\text { h.c. }
$$

where $t_{i j}^{e f f}=t\left\langle\mathbf{n}_{i} \mid \mathbf{n}_{j}\right\rangle, t$ being the bare hopping parameter and $|\mathbf{n}\rangle$ the spin wave function for a spin- $\frac{1}{2}$ quantized along the direction defined by the unit vector $\mathbf{n}=(\sin \theta \cos \phi, \sin \theta \sin \phi, \cos \theta)$. This spinor wave function clearly satisfies (with $\vec{\sigma}=\left(\sigma_{x}, \sigma_{y}, \sigma_{z}\right)$ the vector of Pauli matrices) $\mathbf{n} \cdot \vec{\sigma}|\mathbf{n}\rangle=+|\mathbf{n}\rangle$ and is given by

$$
|\mathbf{n}\rangle=e^{i b}\left(\begin{array}{c}
\cos \frac{\theta}{2} \\
e^{i \phi} \sin \frac{\theta}{2}
\end{array}\right)
$$

where $b$ is an undetermined overall gauge degree of freedom. The effective hopping parameter is then

$$
t_{i j}^{e f f}=t e^{-i\left(b_{i}-b_{j}\right)}\left\{\cos \frac{\theta_{i}}{2} \cos \frac{\theta_{j}}{2}+e^{-i\left(\phi_{i}-\phi_{j}\right)} \sin \frac{\theta_{i}}{2} \sin \frac{\theta_{j}}{2}\right\}
$$

and since $\left|\left\langle\mathbf{n}_{i} \mid \mathbf{n}_{j}\right\rangle\right|^{2}=\cos ^{2} \frac{\theta_{i j}}{2}$, with $\cos \theta_{i j}=\mathbf{n}_{i} \cdot \mathbf{n}_{j}$ or $\theta_{i j}$ being the angle between the two localized spins' directions of quantization so that $\cos ^{2} \frac{\theta_{i j}}{2}=\frac{1}{2}\left(1+\mathbf{n}_{i} \cdot \mathbf{n}_{j}\right)$, we see that we can put

$$
t_{i j}^{e f f}=t e^{i a_{i j}} \cos \frac{\theta_{i j}}{2}
$$

where the Berry phase $a_{i j}$ is obtained (ignoring the gauge parameters) through

$$
e^{i a_{i j}}=\frac{\cos \frac{\theta_{i}}{2} \cos \frac{\theta_{j}}{2}+e^{-i\left(\phi_{i}-\phi_{j}\right)} \sin \frac{\theta_{i}}{2} \sin \frac{\theta_{j}}{2}}{\cos \frac{\theta_{i j}}{2}}
$$

and can be evaluated, e.g., by means of

$$
\sin a_{i j}=-\frac{\sin \frac{\theta_{i}}{2} \sin \frac{\theta_{j}}{2} \sin \left(\phi_{i}-\phi_{j}\right)}{\cos \frac{\theta_{i j}}{2}} .
$$

To see what the phase $a_{i j}$ is, geometrically, we introduce the unit vector $\hat{z}$ and evaluate the triple product

$$
\mathbf{n}_{i} \times \mathbf{n}_{j} \cdot \hat{z}=\sin \theta_{i} \sin \theta_{j} \sin \left(\phi_{i}-\phi_{j}\right)=4 \sin \frac{\theta_{i}}{2} \sin \frac{\theta_{j}}{2} \cos \frac{\theta_{i}}{2} \cos \frac{\theta_{j}}{2} \sin \left(\phi_{i}-\phi_{j}\right)
$$

which shows that

$$
\sin a_{i j}=-\frac{\mathbf{n}_{i} \times \mathbf{n}_{j} \cdot \hat{z}}{4 \cos \frac{\theta_{i}}{2} \cos \frac{\theta_{j}}{2} \cos \frac{\theta_{i j}}{2}} .
$$

This expression is a special case of the formula giving the solid angle $\Omega\left(\mathbf{n}_{1}, \mathbf{n}_{2}, \mathbf{n}_{3}\right)$ between three unit vectors $\mathbf{n}_{1}, \mathbf{n}_{2}$ and $\mathbf{n}_{3}$ :

$$
\sin \frac{\Omega\left(\mathbf{n}_{1}, \mathbf{n}_{2}, \mathbf{n}_{3}\right)}{2}=\frac{\mathbf{n}_{1} \times \mathbf{n}_{2} \cdot \mathbf{n}_{3}}{4 \cos \frac{\theta_{12}}{2} \cos \frac{\theta_{13}}{2} \cos \frac{\theta_{23}}{2}}
$$


(as can be verified by taking, e.g., $\mathbf{n}_{1}=\hat{x}, \mathbf{n}_{2}=\hat{y}$ and $\mathbf{n}_{3}=\hat{z} ; \Omega\left(\mathbf{n}_{1}, \mathbf{n}_{2}, \mathbf{n}_{3}\right)$ can also be seen as the area of the portion of unit sphere enclosed by the maximum circles passing through the unit vectors' tips). In the last formula, of course, $\cos \theta_{k k^{\prime}}=\mathbf{n}_{k} \cdot \mathbf{n}_{k^{\prime}}$ and therefore $\cos ^{2} \frac{\theta_{k k^{\prime}}}{2}=\frac{1}{2}\left(1+\mathbf{n}_{k} \cdot \mathbf{n}_{k^{\prime}}\right)$. We remark that this formula for three spins is completely analogous to that for the chirality gauge field in the formulation of Lee and Nagaosa (1992) for the chiral spin liquid theory of high-temperature superconductivity (Wen, Wilczek and Zee, 1989). In this formulation the instantaneous gauge field flux through the triangular plaquette made up by the three spins is $\Phi\left(\mathbf{n}_{1}, \mathbf{n}_{2}, \mathbf{n}_{3}\right)=\frac{1}{2} \Omega\left(\mathbf{n}_{1}, \mathbf{n}_{2}, \mathbf{n}_{3}\right)$. Back to our two-spins hopping problem, we then conclude that $\sin a_{i j}=-\sin \frac{1}{2} \Omega\left(\mathbf{n}_{i}, \mathbf{n}_{j}, \hat{z}\right)$, or

$$
a_{i j}=\pi+\frac{1}{2} \Omega\left(\mathbf{n}_{i}, \mathbf{n}_{j}, \hat{z}\right)
$$

(the factor $\frac{1}{2}$ being probably due to our specially chosen localised spin value, which leads to the conjecture that for a generic spin- $S$ situation the Berry phase would be $\left.a_{i j}=\pi+S \Omega\left(\mathbf{n}_{i}, \mathbf{n}_{j}, \hat{z}\right)\right)$. Since the solid angle $\Omega\left(\mathbf{n}_{i}, \mathbf{n}_{j}, \hat{z}\right)$ is also the unit sphere's surface area between the tips of the three vectors $\mathbf{n}_{i}, \mathbf{n}_{j}$ and $\hat{z}$, the phase $a_{i j}=\int_{i}^{j} d \mathbf{r} \cdot \mathbf{A}$ can also be seen as the flux of a magnetic monopole's field of modulus $|\mathbf{B}|=\frac{1}{2}$ with the monopole placed in the sphere's center, or, alternatively, as the flux of the related gauge field A through the triangle bearing on the segment $(i, j)$ of a triangular Kagome lattice's unit cell (Ohgushi, Murakami and Nagaosa, 2000). In this way, the Berry phase $a_{i j}$ acquires some physical meaning too.

We now consider this tight-binding model on the Kagome lattice with a fixed localised-spin configuration (or spin texture) as suggested by Ohgushi, Murakami and Nagaosa (2000), in which the unit vectors $\mathbf{n}_{i}$ at each site of a triangular unit cell are tilted outwards at a fixed angle $\theta$ over the unit vector $\hat{z}$ orthogonal to the lattice plane. This means (labelling the spins clockwise A, B and C in the unit cell)

$$
\sin a_{A B}=\sin a_{B C}=\sin a_{C A}=\frac{\sqrt{3} \sin ^{2} \theta}{4(1+\cos \theta) \sqrt{1-\frac{3}{4} \sin ^{2} \theta}} .
$$

The flux generated by the spins in every triangular unit is set equal to $\phi$ with the condition

$$
e^{i \phi}=e^{i\left(a_{A B}+a_{B C}+a_{C A}\right)}=e^{3 i a_{A B}}
$$

with $\phi=3 a_{A B}(\bmod 2 \pi)$ and thus

$$
\sin \frac{\phi}{3}=\frac{\sqrt{3}(1-\cos \theta)}{2 \sqrt{1+3 \cos ^{2} \theta}}
$$

This is equivalent to the expression proposed by Ohgushi, Murakami and Nagaosa (preprint of Ohgushi, Murakami and Nagaosa (2000)) $\phi=\pi+3 \arg (1-i \sqrt{3} \cos \theta)$. The graph for this expression of $\phi=\phi(\theta)$ is shown in Fig. 2 for convenience.

As pointed out by Ohgushi, Murakami and Nagaosa (2000), the flux per triangular unit cell $\phi$ is cancelled out for the chosen spin texture by the flux $-2 \phi$ generated by each of the remaining hexagonal hopping plaquettes on the Kagome lattice. There are indeed twice as many triangular units as hexagonal plaquettes, so that the overall gauge field flux through the lattice is zero. This situation is reminiscent of the analogous tight-binding model in a staggered magnetic field as was proposed by Haldane (1988) to mimick the quantized Hall effect in a condensed-matter situation. In the present model, the chosen spin-texture, with all localised spins tilted by the same angle $\theta$ in each unit cell, is presumably the one corresponding to the mean-field solution for some magnetic spin-spin Heisenberg Hamiltonian which should be added to our tight-binding Hamiltonian, Eq. (11), to give a total Hamiltonian of the type

$$
H_{t o t}=\sum_{\langle i, j\rangle} t_{i j}^{e f f}\left(\left\{\mathbf{S}_{i}^{(0)}\right\}\right) c_{i}^{\dagger} c_{j}+\text { h.c. }+\sum_{i, j} J_{i j} \mathbf{S}_{i} \cdot \mathbf{S}_{j} .
$$

The role of the spin fluctuations around this ordered spin texture, $\left\{\mathbf{S}_{i}^{(0)}\right\}$, as well as the effects of different spin textures, (e.g. AFM ones) could serve as an interesting further research problem for future studies. 


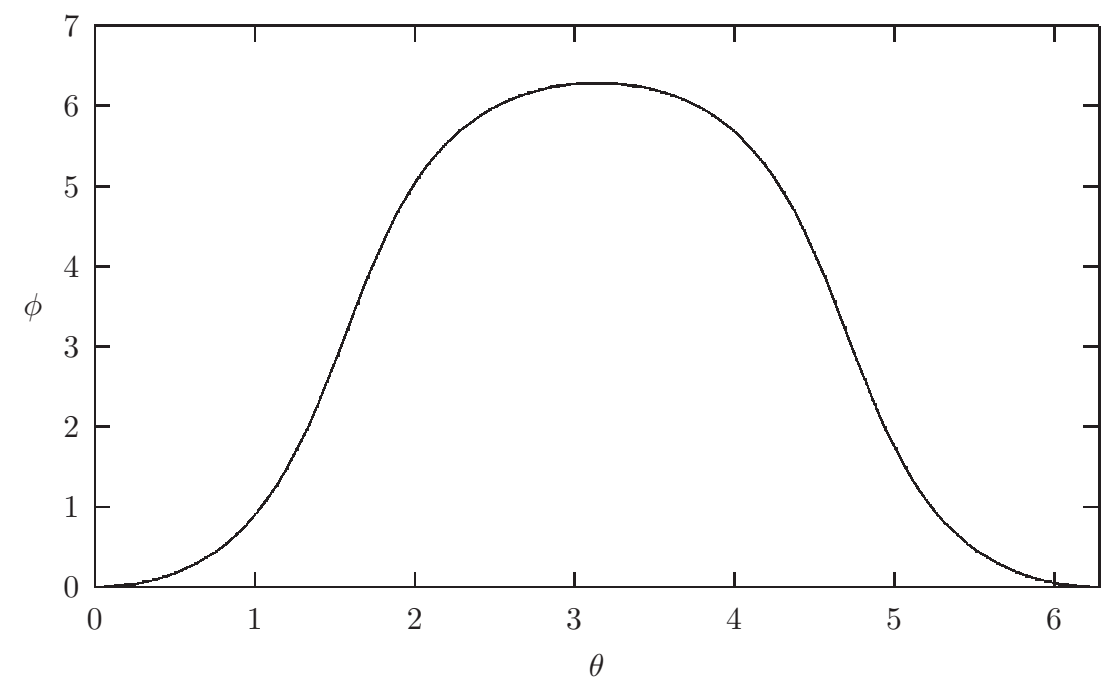

Figure 2: Flux $\phi$ as a function of the tilting angle $\theta$ of the localized spins.

\subsection{Band structure for the Kagome lattice}

The Kagome lattice is made up of triangular and hexagonal plaquettes and can be seen as a triangular lattice with a 3 -point basis where every triangular unit cell contains three sites, $A, B, C$ (Fig 1). The displacement vectors between these sites are $\vec{a}_{1}=(-1 / 2,-\sqrt{3} / 2), \vec{a}_{2}=(1,0)$ and $\vec{a}_{3}=(-1 / 2, \sqrt{3} / 2)$, with $\sum_{i} \vec{a}_{i}=0$. The effective hopping parameter of (1) can be written as

$$
t_{i j}^{e f f}=t e^{i a_{i j}} \cos \left(\frac{\theta_{i j}}{2}\right)
$$

and since $\cos \left(\theta_{i j} / 2\right)=\sqrt{1-\frac{3}{4} \sin ^{2} \theta}$ is fixed for the chosen spin texture, $\theta_{i j}$ being the angle between the n.n. pair of localized spins, we can choose the convention where $t \cos \left(\frac{\theta_{i j}}{2}\right) \equiv 1$. Then, in momentum space, the Hamiltonian can be rewritten as

$$
H=\sum_{\vec{k}} \psi^{\dagger}(\vec{k}) h(\vec{k}) \psi(\vec{k})
$$

where $\psi(\vec{k})=\left(c_{A}(\vec{k}), c_{B}(\vec{k}), c_{C}(\vec{k})\right)$ and $h(\vec{k})$ is the (suitably symmetrized) matrix

$$
h(\vec{k})=\left(\begin{array}{ccc}
0 & 2 \cos \left(\vec{k} \cdot \vec{a}_{1}\right) e^{-i \phi / 3} & 2 \cos \left(\vec{k} \cdot \vec{a}_{3}\right) e^{i \phi / 3} \\
2 \cos \left(\vec{k} \cdot \vec{a}_{1}\right) e^{i \phi / 3} & 0 & 2 \cos \left(\vec{k} \cdot \vec{a}_{2}\right) e^{-i \phi / 3} \\
2 \cos \left(\vec{k} \cdot \vec{a}_{3}\right) e^{-i \phi / 3} & 2 \cos \left(\vec{k} \cdot \vec{a}_{2}\right) e^{i \phi / 3} & 0
\end{array}\right) .
$$

The three eigenvalues of this Hamiltonian are

$$
\begin{aligned}
E_{\text {up }}(\vec{k}) & =4 \sqrt{\frac{1+f(\vec{k})}{3}} \cos \left(\frac{\theta(\vec{k})}{3}\right) \\
E_{\text {mid }}(\vec{k}) & =4 \sqrt{\frac{1+f(\vec{k})}{3}} \cos \left(\frac{\theta(\vec{k})-2 \pi}{3}\right) \\
E_{\text {down }}(\vec{k}) & =4 \sqrt{\frac{1+f(\vec{k})}{3}} \cos \left(\frac{\theta(\vec{k})+2 \pi}{3}\right)
\end{aligned}
$$


with

$$
\theta(\vec{k})=\arg \left[f(\vec{k}) \cos (\phi)+i \sqrt{4\left(\frac{1+f(\vec{k})}{3}\right)^{3}-(f(\vec{k}) \cos (\phi))^{2}}\right]
$$

and

$$
f(\vec{k})=2 \cos \left(\vec{k} \cdot \vec{a}_{1}\right) \cos \left(\vec{k} \cdot \vec{a}_{2}\right) \cos \left(\vec{k} \cdot \vec{a}_{3}\right) .
$$

The three bands touch in six points only for $\phi=0$ and $\phi= \pm \pi$, while for $\phi$ different from these values there is a gap between the bands, as shown in Fig. $3(\phi=0)$ and Fig. $4(\phi=\pi / 3)$.

$$
E(k)
$$

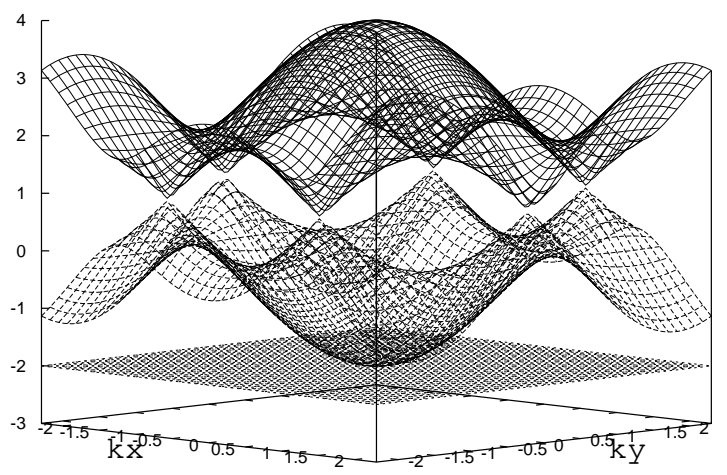

Figure 3: Eigenvalues for the Kagome lattice for $\phi=0$ $E(k)$

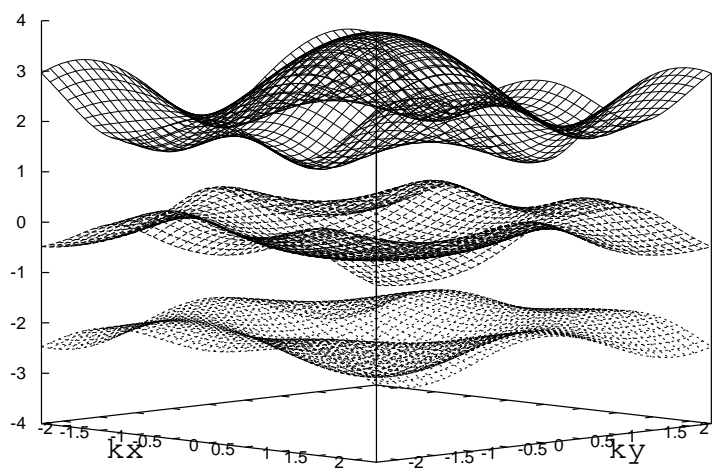

Figure 4: Eigenvalues for the Kagome lattice for $\phi=\pi / 3$

These nodes are: $\left( \pm \frac{2 \pi}{3}, 0\right)$ and $\left( \pm \frac{\pi}{3}, \pm \frac{\sqrt{3}}{3} \pi\right)$, on the vertices of a hexagon, as shown in Fig. 5 .

The problem of calculating transport coefficients with this $3 \times 3$ matrix is not exactly solvable, so we reduce this matrix to a $2 \times 2$ one by expanding $h(\vec{k})$ around the nodes. This can be done with a unitary transformation which allows us to neglect the terms related to the lower band; in fact this band 


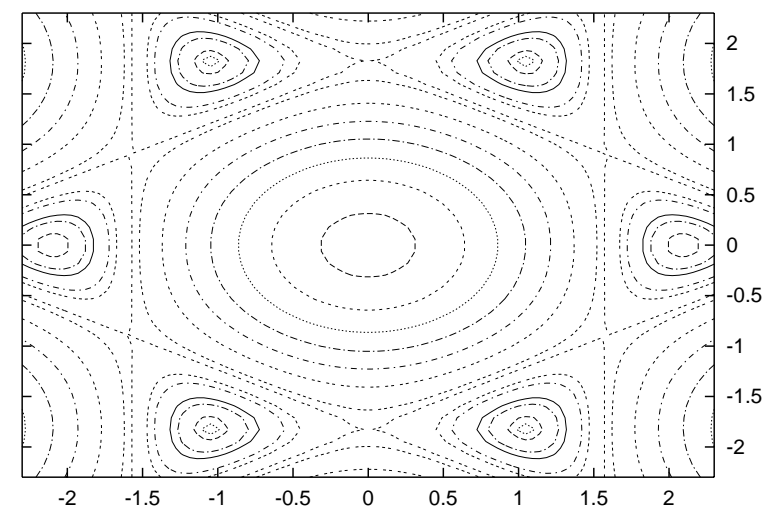

ky

$\mathrm{kx}$

Figure 5: Nodes position for the Kagome lattice

is far from the other two and gives no relevant contribution to the Green function present in the Kubo formula. To find this unitary transformation we consider the Hamiltonian evaluated at a node $\mathbf{k}_{0}$. If we apply the unitary matrix that diagonalizes $h\left(k_{x 0}, k_{y 0}\right)$, where $\left(k_{x 0}, k_{y 0}\right)$ are the node's coordinates, to the Hamiltonian evaluated at the general point $\left(k_{x}, k_{y}\right)$, we find a matrix $H^{\prime}$ with the structure

$$
H^{\prime}=\left(\begin{array}{ccc}
\lambda_{1} & \alpha\left[\tilde{k}_{x}-i \tilde{k}_{y}\right] & \beta\left[\tilde{k}_{x}-i \tilde{k}_{y}\right] \\
\bar{\alpha}\left[\tilde{k}_{x}+i \tilde{k}_{y}\right] & \lambda_{2} & \gamma\left[\tilde{k}_{x}+i \tilde{k}_{y}\right] \\
\bar{\beta}\left[\tilde{k}_{x}+i \tilde{k}_{y}\right] & \bar{\gamma}\left[\tilde{k}_{x}-i \tilde{k}_{y}\right] & \lambda_{3}
\end{array}\right)
$$

with $\tilde{k}_{x}=\left(k_{x}-k_{x 0}\right)$ and $\tilde{k}_{y}=\left(k_{y}-k_{y 0}\right)$. The elements on the diagonal are the eigenvalues of the Hamiltonian $h\left(k_{x 0}, k_{y 0}\right)$, while the off-diagonal elements are complex combinations of $\tilde{k}_{x, y}$. Near the nodes the distance between the upper and the middle band is small, while the lower band is distant and gives no relevant contribution. To justify this we can consider a projection of the Green function around the node.

We want to find a projection only on the first two eigenvalues, so we choose a projector such that

$$
P H^{\prime} P=\left(\begin{array}{cc}
\lambda_{1} & H_{12}^{\prime} \\
H_{21}^{\prime} & \lambda_{2}
\end{array}\right)
$$

We define the Green function as $G=\left(z-H^{\prime}\right)^{-1}$ and the projection operator $P$ with the convention that $(A)_{P}^{-1}=(P A P)^{-1}$ is the inverse operation on the projected space, $(1-P)$ being the projection operator complementary to $P$. The Green function can now be written as

$$
G=P G P+(1-P) G P+P G(1-P)+(1-P) G(1-P)
$$

and the projected Green function is

$$
P G P=P\left(z-H^{\prime}\right)^{-1} P=\left(z-P H^{\prime} P-P H^{\prime}(1-P)\left(z-H^{\prime}\right)_{1-P}^{-1}(1-P) H^{\prime} P\right)_{P}^{-1} .
$$

The last terms are of higher order and can be neglected. If we consider the terms related to the lower eigenvalue, we can see that $1 /\left(z-\lambda_{3}\right) \sim 1 / 2$, because we are considering $\vec{k}$ near the nodes. This eigenvalue 
does not give an important contribution, so it can be neglected and we can use the approximation

$$
P G P \approx\left(z-P H^{\prime} P\right)_{P}^{-1} .
$$

Now we can write the projection of the Hamiltonian $H^{\prime}$ as

$$
\begin{aligned}
P H^{\prime} P & =\left(\begin{array}{cc}
\lambda_{1} & \alpha\left[\tilde{k}_{x}-i \tilde{k}_{y}\right] \\
\bar{\alpha}\left[\tilde{k}_{x}+i \tilde{k}_{y}\right] & \lambda_{2}
\end{array}\right) \\
& =\left(\begin{array}{cc}
1+\frac{\sqrt{3} \phi}{3} & h_{1}-i h_{2} \\
h_{1}+i h_{2} & 1-\frac{\sqrt{3} \phi}{3}
\end{array}\right) \\
& =I+\left(\begin{array}{cc}
m & h_{1}-i h_{2} \\
h_{1}+i h_{2} & -m
\end{array}\right)=I+h
\end{aligned}
$$

where $I$ is the identity matrix, with

$$
\begin{aligned}
h_{1} & =\alpha_{1} \tilde{k}_{x}+\alpha_{2} \tilde{k}_{y} \\
h_{2} & =-\alpha_{2} \tilde{k}_{x}+\alpha_{1} \tilde{k}_{y} \\
m & =\frac{\sqrt{3}}{3} \phi,
\end{aligned}
$$

with $\alpha_{1}$ and $\alpha_{2}$ are the components of a complex parameter, depending on the node we are considering. The new matrix representing the Hamiltonian has eigenvalues $\pm \lambda$ and eigenvectors $\Psi_{ \pm}$with

$$
\begin{aligned}
\lambda & =\sqrt{m^{2}+h_{1}^{2}+h_{2}^{2}} \\
\Psi_{ \pm} & =\frac{1}{\sqrt{1+( \pm \lambda-m)^{2} /|k|^{2}}}\left(\begin{array}{c}
1 \\
\frac{ \pm \lambda-m}{k}
\end{array}\right) .
\end{aligned}
$$

As was said, the bands touch only when $\phi=0$ and $\phi= \pm \pi$. Here we consider $\phi$ different from zero, but small enough so that we create a small gap between the two bands.

\subsection{Band structure of the honeycomb lattice}

Here we also consider the case of a honeycomb lattice, as was first envisaged by Haldane (1988); this is made up by two sublattices that we call $\mathrm{A}$ and $\mathrm{B}$ (Fig. 6), or by a triangular lattice with a 2-point basis. The displacement vectors from a $B$ site to the three nearest neighbours are: $\vec{a}_{1}=(-\sqrt{3} / 2,1 / 2)$, $\vec{a}_{2}=(0,-1)$ and $\vec{a}_{3}=(\sqrt{3} / 2,1 / 2)$, while the displacement vectors from the site $\mathrm{B}$ and the nearest neighbours on the same sublattice are $\vec{b}_{1}=(-\sqrt{3} / 2,-3 / 2), \vec{b}_{2}=(\sqrt{3}, 0)$ and $\vec{b}_{3}=(-\sqrt{3} / 2,3 / 2)$ (again, $\left.\sum \vec{b}_{i}=0\right)$.

Here too, we consider a tight-binding model in the presence of a staggered magnetic flux (Haldane, 1988); the Hamiltonian for this Haldane model is $H=\sum_{\vec{k}} \psi^{\dagger}(\vec{k}) h(\vec{k}) \psi(\vec{k})$ with

$$
\begin{aligned}
h(\vec{k}) & =\quad 2 t_{2} \cos \phi \sum_{i} \cos \left(\mathbf{k} \cdot \mathbf{b}_{\mathbf{i}}\right) \mathbf{I}+t_{1} \sum_{i}\left[\cos \left(\mathbf{k} \cdot \mathbf{a}_{\mathbf{i}}\right) \sigma_{\mathbf{1}}+\sin \left(\mathbf{k} \cdot \mathbf{a}_{\mathbf{i}}\right) \sigma_{\mathbf{2}}\right] \\
& +\quad\left[M-2 t_{2} \sin \phi \sum_{i} \sin \left(\mathbf{k} \cdot \mathbf{b}_{\mathbf{i}}\right)\right] \sigma_{\mathbf{3}},
\end{aligned}
$$

where $t_{1}$ is a hopping parameter between nearest neighbours on different sublattices, $t_{2}$ is a hopping parameter between nearest neighbour sites on the same sublattice, and $\sigma_{i}$ are the three Pauli matrices. If we rewrite the Hamiltonian as $H=a \sigma_{1}+b \sigma_{2}+c \sigma_{3}$, the Hamiltonian matrix reads

$$
H=\left(\begin{array}{cc}
c & a-i b \\
a+i b & -c
\end{array}\right) .
$$




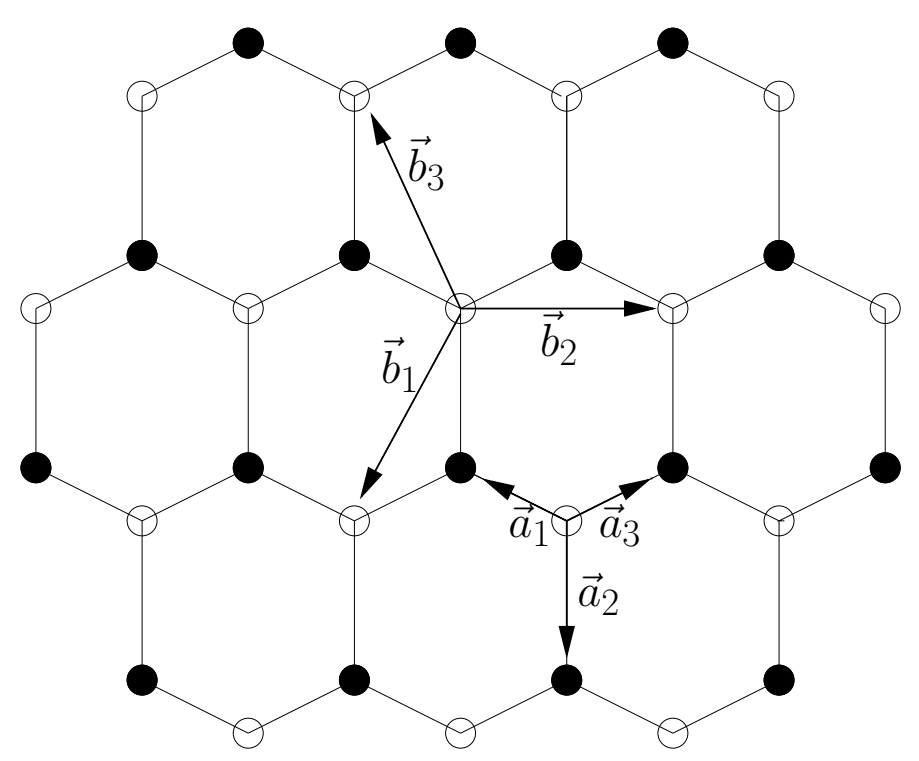

Figure 6: Honeycomb lattice

The eigenvalues of this matrix are $\pm \lambda$ with $\lambda=\sqrt{a^{2}+b^{2}+c^{2}}$ (Fig. $7(\phi=0)$ and Fig. $\left.8\left(\phi=\frac{\pi}{3}\right)\right)$, while the eigenvectors are

$$
\psi_{ \pm}=\frac{1}{\sqrt{1+\frac{( \pm \lambda)^{2}}{|k|^{2}}}}\left(\begin{array}{c}
1 \\
\pm \lambda-c
\end{array}\right)
$$

where $k=a-i b$. Formally this case is similar to that of the Kagome lattice, after the reduction of the original matrix to a $2 \times 2$ one. The two bands meet when the condition $a^{2}+b^{2}+c^{2}=0$ is satisfied. This becomes a condition on the parameter $M$ : there are nodes when $M=3 \sqrt{3} t_{2} \alpha \sin \phi$, with $\alpha= \pm 1$. When $\phi=0$ and $M=0$ there are six nodes: $\left( \pm \frac{4 \pi}{3 \sqrt{3}}, 0\right)$ and $\left( \pm \frac{2 \pi}{3 \sqrt{3}}, \pm \frac{2 \pi}{3}\right)$ (Fig. 9), while when $M=3 \sqrt{3} t_{2} \alpha \sin \phi$ there are only three of these nodes.

\section{$3 \quad$ Transport Properties}

Based on the linear-response theory, a suitable Kubo formula and the corresponding conductivity tensor can be studied for the Hamiltonians considered in Sect. 2. Some details are given in Appendix A. From this result we can derive the Hall conductivity $\sigma_{x y}$ and the longitudinal conductivity $\sigma_{x x}$ of our two-dimensional tight-binding model. We verify explicitely the quantization of $\sigma_{x y}$ as a function of $\phi$ (or $\theta$ ) and calculate explicitely the longitudinal conductivity $\sigma_{x x}$ which also appears to be quantized in the absence of disorder or other symmetry-breaking conditions. For the two models considered, these are our main new results.

\subsection{Hall conductivity}

For $\mu \neq \nu$ the third term in Eq. (62) (Appendix A) vanishes and after the integration with respect to $E$ we find that the Hall conductivity, in the limit of $\omega=0$ and $T=0$, is

$$
\sigma_{x y}=-\frac{1}{\hbar \eta} R e \sum_{k} \lambda_{k}\left[U^{\dagger} j_{\mu}\left(h-\lambda_{k}+2 i \eta\right)^{-1} j_{\nu} U\right]_{k k},
$$

where $U$ is the unitary matrix that diagonalizes the Hamiltonian matrix $h(\vec{k})$, while $j_{x}$ and $j_{y}$ are the current matrices. From this we find that the Hall conductivity for every node $n$ for the case of the Kagome 


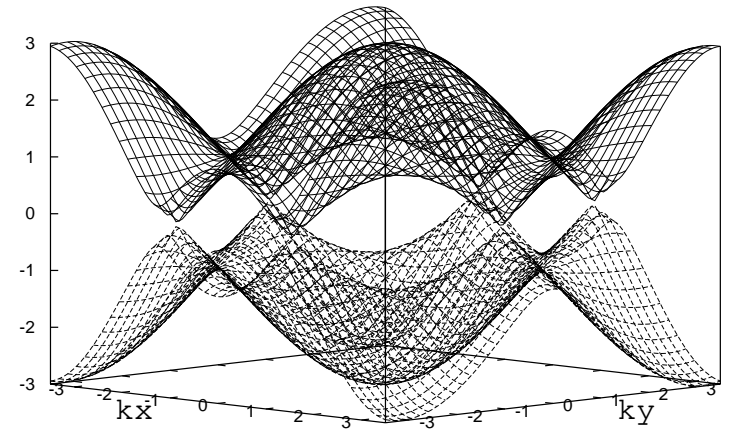

Figure 7: Eigenvalues for the honeycomb lattice for $\phi=0$

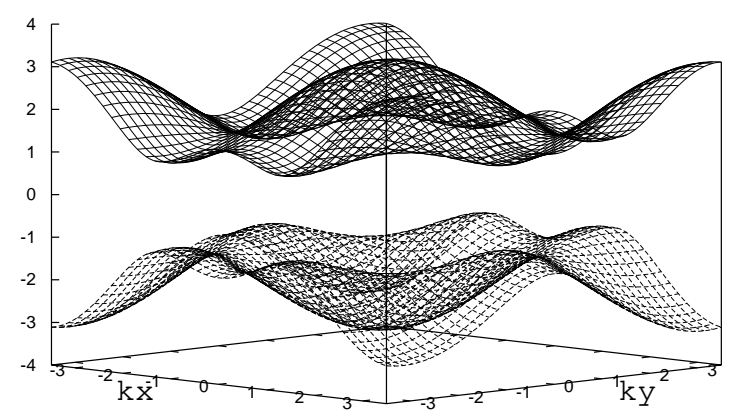

Figure 8: Eigenvalues for the honeycomb lattice for $\phi=\frac{\pi}{3}, M \neq 0$ and $M \neq 3 \sqrt{3} \alpha t_{2} \sin \phi$

lattice is

$$
\sigma_{x y}^{n}=\frac{e^{2}}{\hbar \eta} \int_{-\infty}^{+\infty} \frac{12 m \eta}{8\left(m^{2}+h_{1}^{2}+h_{2}^{2}\right)^{\frac{3}{2}}} \frac{d^{2} k}{(2 \pi)^{2}}=\frac{e^{2}}{2 h} \operatorname{sgn}(\phi) .
$$

The integration being over the hexagonal Brillouin zone, we considered only one third of the integral and then we have to multiply for the number of nodes. We can conclude that the Hall conductivity is different from zero and it is quantized in the presence of a gap between the bands (that is for $\phi$ different from 0 , $\pm \pi)$ and is equal to

$$
\sigma_{x y}=\frac{e^{2}}{h} \operatorname{sgn}(\phi)
$$

So, we have another model of transverse conductivity quantization in the absence of an external uniform magnetic field.

Now we consider the case of the honeycomb lattice, in Haldane's model. For $M=0$ and $\phi=0$ the bands touch in six points: $\left( \pm \frac{4 \pi}{3 \sqrt{3}}, 0\right),\left( \pm \frac{2 \pi}{3 \sqrt{3}}, \pm \frac{2 \pi}{3}\right)$ and the Hamiltonian is simply of the form

$$
\left(\begin{array}{cc}
0 & a-i b \\
a+i b & 0
\end{array}\right)
$$




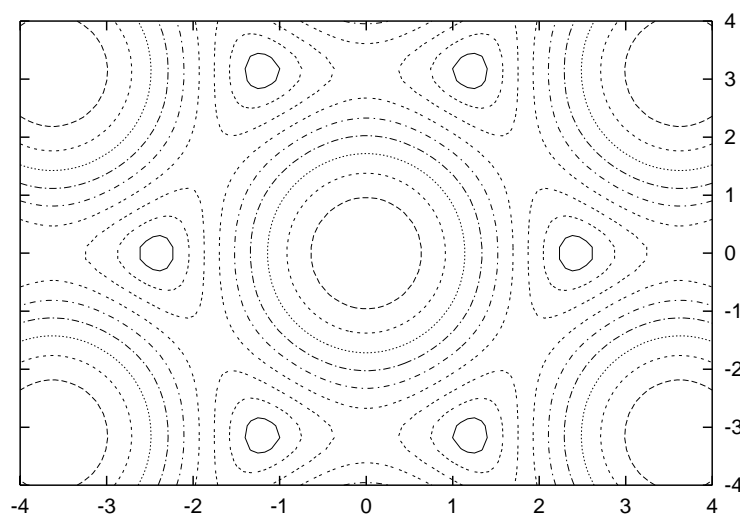

ky

$\mathrm{kx}$

Figure 9: Nodes position for the honeycomb lattice for $\phi=0$

Expanding the terms around the nodes, we find that the function to integrate in order to find the Hall conductivity is

$$
\pm \frac{9 a b}{8\left(a^{2}+b^{2}\right)^{\frac{3}{2}}}
$$

but the integral of this term gives zero contribution. To generate a gap we have to move from the situation in which $M=0$ and $\phi=0$. We add a small mass contribution $M \ll 1$, but we mantain $\phi=0$; now the Hamiltonian is

$$
\left(\begin{array}{cc}
M & a-i b \\
a+i b & -M
\end{array}\right)
$$

In this case the function to integrate is

$$
\pm \frac{9 t_{1}^{2} M \eta}{8\left(a^{2}+b^{2}+M^{2}\right)^{\frac{3}{2}}}
$$

three nodes give a positive contribution and three a negative one, to give

$$
\pm \frac{e^{2}}{2 h} \operatorname{sgn}(M)
$$

Summing up all the contributions we find that the Hall conductivity is zero. Different is the situation in which we consider $M=0$, but we add a small flux $\phi$. We rewrite the Hamiltonian as

$$
\left(\begin{array}{cc}
c & a-i b \\
a+i b & -c
\end{array}\right)
$$

where

$$
c=-2 t_{2} \sin \phi \sum_{i} \sin \left(\mathbf{k} \cdot \mathbf{b}_{\mathbf{i}}\right)
$$


Now the function to integrate is $(k=a-i b)$

$$
P(k)= \pm \frac{9 c \eta t_{1}^{2}}{8\left(a^{2}+b^{2}+c^{2}\right)^{\frac{3}{2}}} .
$$

Near three of the six nodes $P(k)$ is negative, but approximating $c$ around these points we find $c \simeq$ $3 \sqrt{3} t_{2} \sin \phi$, so the function to integrate is negative. After the integration we find that every one of these three points gives a conductivity equal to

$$
\frac{e^{2}}{2 h} \operatorname{sgn}(\sin \phi)
$$

For the other three points $P(k)$ is positive, but the expansion of $c$ is $c \simeq-3 \sqrt{3} t_{2} \sin (\phi)$. So, now the function to integrate is negative too and it gives the same result as before. Summing over all the points and remembering that the integration is over the exagon we find that the Hall conductivity for the honeycomb lattice case is

$$
\sigma_{x y}=\frac{e^{2}}{h} \operatorname{sgn}(\sin \phi) .
$$

We can conclude that the Hall conductivity can be rewritten as

$$
\sigma_{x y}=\nu \frac{e^{2}}{h}
$$

with $\nu= \pm 1$, depending on the sign of $\phi$.

\subsection{Longitudinal conductivity}

Here too we use the general expression derived from the Kubo formula. In this case $(\mu=\nu=x)$ the longitudinal conductivity $\sigma_{x x}$ derived by expression (62), after the energy integration, is

$$
-\frac{1}{\hbar} \sum_{k, m} \frac{\rho_{0}\left(\lambda_{k}\right)\left(U^{\dagger} j_{x} U\right)_{k m}\left(U^{\dagger} j_{x} U\right)_{m k}}{-\omega+2 i \eta}\left[\frac{1}{\lambda_{m}-\lambda_{k}-\omega+2 i \eta}+\frac{1}{\lambda_{m}-\lambda_{k}+\omega-2 i \eta}\right] .
$$

After summing over the eigenvalues, substituting the values of $\lambda_{k}$ and $\epsilon=\eta+\frac{i \omega}{2}$ we find

$$
\sigma_{x x}=-\frac{1}{2 \hbar \epsilon} \int_{-\infty}^{+\infty}\left(U^{\dagger} j_{x} U\right)_{21}\left(U^{\dagger} j_{x} U\right)_{12} \frac{\sqrt{m^{2}+h_{1}^{2}+h_{2}^{2}}}{m^{2}+h_{1}^{2}+h_{2}^{2}+\epsilon^{2}} \frac{d^{2} k}{(2 \pi)^{2}} .
$$

$>$ From this expression, we have still to subtract the diamagnetic term and so we evaluate (Ludwig, Fisher, Shankar and Grinstein, 1994)

$$
\tilde{\sigma}_{x x}=\sigma_{x x}-\frac{1}{\epsilon} \lim _{\epsilon \rightarrow 0} \epsilon \sigma_{x x} .
$$

For the Kagome lattice the product of the matrix elements of the currents is

$$
\left(U^{\dagger} j_{x} U\right)_{21}\left(U^{\dagger} j_{x} U\right)_{12}=\frac{3\left(4 m^{2}+3 h_{1}^{2}+2 \sqrt{3} h_{1} h_{2}+h_{2}^{2}\right)}{4\left(m^{2}+h_{1}^{2}+h_{2}^{2}\right)},
$$

and remembering that $h_{1}$ and $h_{2}$ are symmetric variables and using polar coordinates we can rewrite $\sigma_{x x}$ as

$$
\sigma_{x x}=-\frac{e^{2}}{4 h \epsilon}\left[\int_{0}^{\infty} \frac{m^{2} r}{\sqrt{m^{2}+r^{2}}\left(m^{2}+r^{2}+\epsilon^{2}\right)} d r+\int_{0}^{\infty} \frac{r \sqrt{m^{2}+r^{2}}}{m^{2}+r^{2}+\epsilon^{2}} d r\right],
$$

so that carrying out the integrals we find

$$
\int_{0}^{\infty} \frac{r m^{2}}{\epsilon \sqrt{m^{2}+r^{2}}\left(m^{2}+r^{2}+\epsilon^{2}\right)} d r= \begin{cases}\frac{m^{2}}{\epsilon^{2}} \arccos \left(\frac{m}{\sqrt{m^{2}+\epsilon^{2}}}\right) & \text { if } m>0 \\ 0 & \text { if } m=0 \\ \frac{m^{2}}{\epsilon^{2}}\left(\pi-\arccos \left(\frac{m}{\sqrt{m^{2}+\epsilon^{2}}}\right)\right. & \text { if } m<0\end{cases}
$$


For the second integral we introduce a cut-off $\lambda$ and evaluate

$$
\int_{0}^{\lambda} \frac{r \sqrt{m^{2}+r^{2}}}{\epsilon\left(m^{2}+r^{2}+\epsilon^{2}\right)} d r= \begin{cases}\frac{\lambda}{\epsilon}\left(-m+\sqrt{m^{2}+\lambda^{2}}\right)+\arctan \left(\frac{m}{\epsilon}\right)-\arctan \left(\frac{\sqrt{m^{2}+\lambda^{2}}}{\epsilon}\right) & \text { if } m>0 \\ \frac{\lambda}{\epsilon}-\arctan \left(\frac{\lambda}{\epsilon}\right) & \text { if } m=0 \\ \frac{\lambda}{\epsilon}\left(m+\sqrt{m^{2}+\lambda^{2}}\right)-\arctan \left(\frac{m}{\epsilon}\right)-\arctan \left(\frac{\sqrt{m^{2}+\lambda^{2}}}{\epsilon}\right) & \text { if } m<0 .\end{cases}
$$

We consider two cases: $m$ equal to zero (that is, the flux $\phi$ is zero) and $m$ different from zero. In the first case, after having removed the diamagnetic term, the conductivity is

$$
\tilde{\sigma}_{x x}=\frac{e^{2}}{4 h} \arctan \left(\frac{\lambda}{\epsilon}\right) .
$$

Now we can take the limit for $\epsilon \rightarrow 0$ (thus making the cutoff irrelevant). We find that the conductivity for every node is different from zero and is equal to

$$
\tilde{\sigma}_{x x}=\frac{1}{3} \frac{e^{2}}{4 h} \frac{\pi}{2}
$$

After the sum over all six nodes is done we can conclude that the longitudinal conductivity for $m=0$ is

$$
\tilde{\sigma}_{x x}=\frac{e^{2} \pi}{4 h} .
$$

The case where $m$ is not zero is quite different. Now, after having done the diamagnetic subtraction (45), the conductivity is

$$
\tilde{\sigma}_{x x}=\frac{e^{2}}{4 h}\left[\frac{m^{2}}{\epsilon} \arccos \left(\frac{m}{\sqrt{m^{2}+\epsilon^{2}}}\right)-\frac{m}{\epsilon}+\arctan \left(\frac{m}{\epsilon}\right)-\arctan \left(\frac{\sqrt{m^{2}+\lambda^{2}}}{\epsilon}\right)\right],
$$

but the limit for $\epsilon \rightarrow 0$ gives a vanishing result. We conclude that the longitudinal conductivity is different from zero only when there is no gap between the two bands, that is in our case for $m=0$.

Next we consider the longitudinal conductivity for the honeycomb lattice. We expect that it is different from zero when the bands touch. This happens in six points, when $M=0$ and $\phi=0$, and in three points when $M=3 \sqrt{3} \alpha t_{2} \sin (\phi)$. To calculate $\sigma_{x x}$, we use the expression (44). In the first case every node gives a contribution different from zero and equal to each other, so with the same observations made for the Kagome lattice we find that the longitudinal conductivity is

$$
\sigma_{x x}=\frac{e^{2} \pi}{4 h} \quad(M=\phi=0) .
$$

When $M>0$ and $\phi=0$ we find that the conductivity is

$$
\begin{aligned}
\sigma_{x x}= & -\frac{1}{2 \hbar \epsilon} \frac{9}{8} \frac{1}{(2 \pi)^{2}} \int_{-\infty}^{\infty} \frac{\sqrt{h_{1}^{2}+h_{2}^{2}+M^{2}}}{h_{1}^{2}+h_{2}^{2}+M^{2}+\epsilon^{2}} d^{2} k \\
& -\frac{1}{2 \hbar \epsilon} \frac{9}{8} \frac{1}{(2 \pi)^{2}} \int_{-\infty}^{\infty} \frac{M^{2}}{\sqrt{h_{1}^{2}+h_{2}^{2}+M^{2}}\left(h_{1}^{2}+h_{2}^{2}+M^{2}+\epsilon^{2}\right)} d^{2} k
\end{aligned}
$$

but, as in the case of the Kagome lattice, this integral vanishes; so in this case the longitudinal conductivity is zero.

The last case we consider is for $M= \pm 3 \sqrt{3} \alpha t_{2} \sin (\phi) \neq 0$. Here the bands touch only in three points and these give a contribution to the conductivity since now there is a gap where before there were three nodes. Around the three nodes the term $c$ is zero, so formally the problem is the same as that of the case $M=0$ and $\phi=0$ and only in these three points the longitudinal conductivity is different from zero. The result is equal to half of what was found in the case in which $M$ and $\phi$ are zero, that is

$$
\sigma_{x x}=\frac{e^{2} \pi}{8 h} \quad\left(M= \pm 3 \sqrt{3} \alpha t_{2} \sin (\phi) \neq 0\right) .
$$




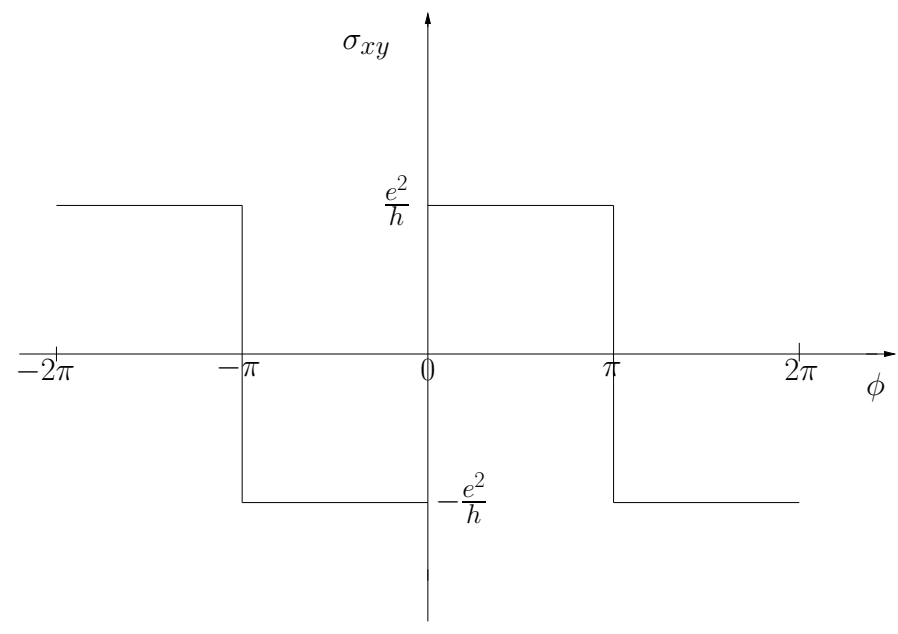

Figure 10: Hall conductivity for electrons on the Kagome lattice.

We can conclude that the longitudinal conductivity can be rewritten as

$$
\sigma_{x x}=\mu \frac{e^{2} \pi}{8 h}
$$

with $\mu=0,1,2$ for the honeycomb lattice and $\mu=0,2$ for the Kagome lattice.

\section{Discussion and Conclusions}

We have considered both Haldane's model for electrons in a staggered flux on the honeycomb lattice and the model by Ohgushi, Murakami and Nagaosa for electrons in the presence of a canted spin- $1 / 2$ texture on the Kagome lattice. We have shown how similar these two models are in that the transverse Hall conductivity $\sigma_{x y}$ is quantized as $\pm e^{2} / h$ as a function of the tuning parameter (e.g. the magnetic flux per plaquette, $\phi)$.

Whilst for the Hall conductivity $\sigma_{x y}$ we have obtained the same results both for the Kagome lattice in the presence of a spin texture (as found by Oshgushi et al. (2000)) and for the honeycomb lattice with staggered magnetic field (as found by Haldane (1988)), we stress that we have used a different method of calculation based on implementing the band structure of each model in the Kubo formula. Furthermore, we have explicitely evaluated in this way, and for the first time, also the longitudinal conductivity $\sigma_{x x}$ starting from the Kubo formula. This quantity is also quantized in the absence of symmetry-breaking, non-ideal features of the system, but not in terms of integer multiples of $e^{2} / h$.

For the Kagome lattice model, we find metallic behavior for a ferromagnetic state of localized spins perpendicular to the plane of the lattice. This state has a vanishing flux $(\phi=0)$ in each plaquette of the Kagome lattice. Metallic behavior exists also for a canted state where the spins are inside the plane $(\theta=\pi / 2$, in this case the local flux is $\phi=\pi)$. The longitudinal conductivity is for both cases $\sigma_{x x}=e^{2} \pi / 4 h$ and the Hall conductivity vanishes. In Fig. 10 we show the schematic behaviour of the Hall conductivity for the model defined on the Kagome lattice and as a function of the parameter $\phi$. This is to be compared with the richer phase diagram for the Haldane model on the honeycomb lattice, reported in Fig. 11 also as a function of $\phi$. The longitudinal conductivity $\sigma_{x x}$ as evaluated in this work is shown schematically in Fig. 12.

Removing some of the nodes in the DOS by breaking symmetries (like for the case of a square lattice with next-nearest neighbor terms) alters the Hall conductivity substantially. Also the introduction of disorder (e.g. slow fluctuations of the localized spins, fluctuations around the perfect canted spin texture) may remove some of the nodes and yield non-universal features in the transport properties. There is also another interesting effect due to disorder in our two-dimensional lattices. The longitudinal conductivity 


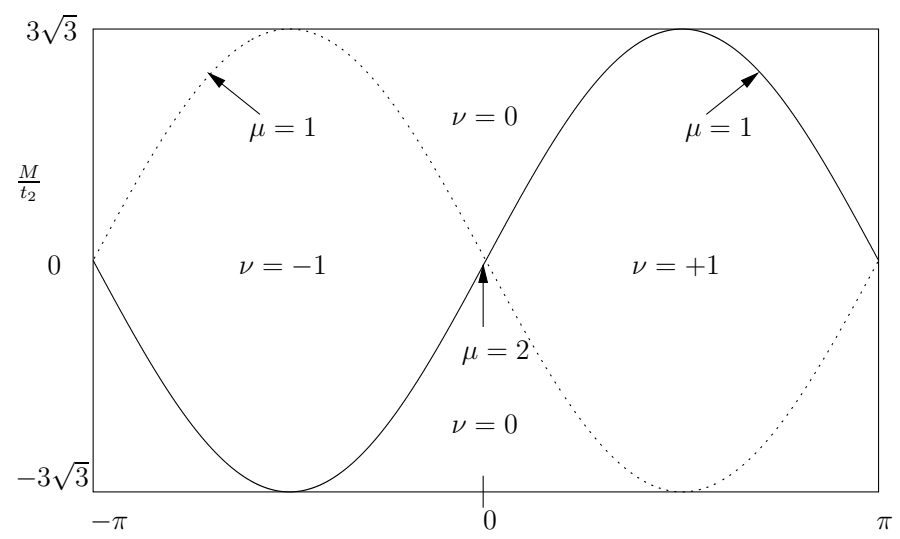

Figure 11: Hall conductivity on the honeycomb lattice as a function of $\phi$; the parameter $\nu$ quantizes $\sigma_{x y}$ in units of $e^{2} / h$ and $\mu$ quantizes $\sigma_{x x}$ in units of $e^{2} \pi / 8 h$.

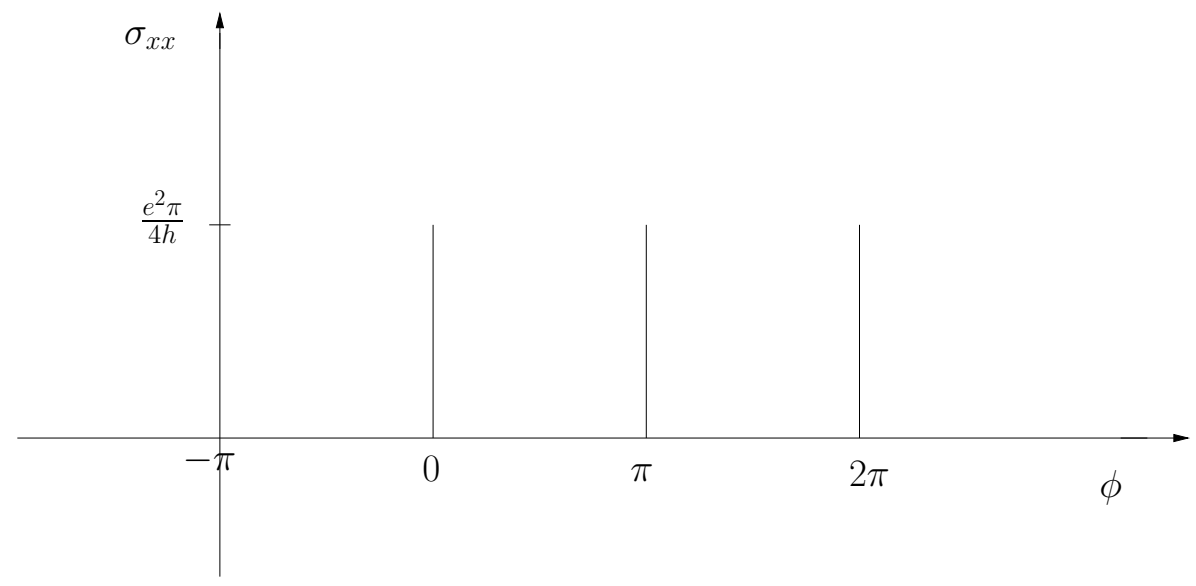

Figure 12: Longitudinal conductivity for electrons on the Kagome lattice. 
$\sigma_{x x}$ is usually based on diffusion of charge carriers; however, the diffusion coefficient $D$ is infinite in our model, since there is no scattering in the absence of imperfections. Nevertheless, the longitudinal conductivity, expressed through the Einstein relation

$$
\sigma_{x x}=\frac{e^{2}}{\hbar} D \rho,
$$

is finite thanks to a vanishing density of states at the nodes. The cancellation of the divergent diffusion coefficient and the vanishing density of states is subtle. Since there is scattering by impurities in a realistic system, a finite diffusion coefficient is more natural. On the other hand, impurities create additional states near the nodes such that a non-vanishing density of states exists. This effect was studied in the case of 2D Dirac fermions with random scatterers (Ziegler, 1997, 1998, Ziegler and Jug, 1997). In particular, it was found that random scattering broadens the metallic state (Ziegler and Jug, 1997), and the maximal conductivity value is lowered by a factor $1 /(1+g / 2 \pi)$, where $g$ is the strength of the random fluctuations.

Acknowledgement:

We are grateful to MIUR (Ministero dell'Istruzione, Universita' e della Ricerca) for support through PRIN-2003 grant and to the Deutsche Forschungsgemeinschaft for support through Sonderforschungsbereich 484 .

\section{Appendix A: Linear Response and Kubo Formula}

From the Kubo formula we know that the conductivity tensor can be written as (Madelung, 1978)

$$
\sigma_{\mu \nu}=\frac{e}{i \hbar} \lim _{\alpha \rightarrow 0} \int_{-\infty}^{0} e^{(i \omega+\alpha) t} \operatorname{Tr}\left(\left[\rho_{0}, r_{\mu}\right] e^{-i H t / \hbar} j_{\nu} e^{i H t / \hbar}\right) d t
$$

where $\rho_{0}$ is the Fermi function. Using the Green functions defined as

$$
G_{ \pm}(E)=(H / \hbar+E \pm i \eta)^{-1}
$$

we can use the substitution

$$
e^{ \pm i H t / \hbar}= \pm \lim _{\eta \rightarrow 0} \int_{-\infty}^{\infty} e^{\mp i E t} G_{\mp}(E) \frac{d E}{2 \pi i} \quad(t \leq 0)
$$

the conductivity can be rewritten as

$$
\sigma_{\mu \nu}=\frac{e}{\hbar} \int_{-\infty}^{\infty} \operatorname{Tr}\left[\rho_{0}, r_{\mu}\right] G_{+}(E) j_{\nu} G_{-}(E+\omega) \frac{d E}{2 \pi i}
$$

The current operator is

$$
j_{\nu}=\frac{e}{i}\left[H, r_{\nu}\right]
$$

Using this expression iteratively, we find that the conductivity can be rewritten as a sum of three terms

$$
\begin{array}{r}
\sigma_{\mu \nu}=-\frac{1}{\hbar} \int_{-\infty}^{\infty} \operatorname{Tr}\left\{\rho_{0} G_{+}(E) j_{\mu} G_{+}(E) j_{\nu} G_{-}(E+\omega)\right\} \frac{d E}{2 \pi} \\
-\frac{1}{\hbar} \int_{-\infty}^{\infty} \operatorname{Tr}\left\{\rho_{0} G_{+}(E) j_{\nu} G_{-}(E+\omega) j_{\mu} G_{-}(E+\omega)\right\} \frac{d E}{2 \pi} \\
+\frac{e}{\hbar} \int_{-\infty}^{\infty} \operatorname{Tr}\left\{\rho_{0} G_{+}(E)\left[j_{\nu}, r_{\mu}\right] G_{-}(E+\omega)\right\} \frac{d E}{2 \pi}
\end{array}
$$




\section{References}

[1] Anderson P.W. and Hasegawa H. 1955, Phys. Rev. 100, 675

[2] de Gennes P.-G. 1960,Phys. Rev. 118, 141

[3] Haldane F.D.M. 1988, Phys. Rev. Lett. 61, 2015

[4] Harris M.J. and Zinkin M.P. 1996, Mod. Phys. Lett. B 10, 417

[5] Katsufuji T., Hwang H.Y. and Cheong S.-W. 2000 Phys. Rev. Lett. 84, 1998

[6] Lee P. and Nagaosa N. 1992, Phys. Rev. B 46, 5621

[7] Ludwig A.W.W. , Fisher M.P.A., Shankar R., Grinstein G. 1994, Phys. Rev. B 50, 7526

[8] Madelung O. 1978, Introduction to Solid-State Theory (Springer-Verlag, Berlin )

[9] Müller-Hartmann E. and Dagotto E. 1996, Phys. Rev. 54, R6819

[10] Ohgushi K., Murakami S. and Nagaosa N. 2000, Phys. Rev. B 62, R6065; see also: ibid. cond-mat/9912206

[11] Ramirez A.P. 1994, Ann. Rev. Mater. Sci. 24453

[12] Taguchi Y. and Tokura Y. 1999, Phys. Rev. B 60, 10280

[13] Wen X.-G., Wilczek F. and Zee A. 1989, Phys. Rev. B 39, 1141

[14] Zener C. 1951, Phys. Rev. 82, 403

[15] Ziegler K. 1997, Phys. Rev. B 55, 10661

[16] Ziegler K.1998, Phys. Rev. Lett. 80, 3113

[17] Ziegler K. and Jug G. 1997, Z. Physik B 104, 5 